Check for updates

Cite this: RSC Adv., 2018, 8, 27963

\title{
Valorization of papaya fruit waste through low-cost fractionation and microbial conversion of both juice and seed lipids $\uparrow$
}

\begin{abstract}
Zhenlin Han, Alex Park and Wei Wen Su (D) *
Papaya (Carica papaya) is widely cultivated in many tropical regions of the world. With an estimated $30-50 \%$ cull rate, there is a large amount of off-grade papaya produced. Here, we report very low-cost processing of culled papaya fruit waste, without needing any complex mechanized operations, to yield several products, including seed oil, sugar-rich puree, detoxified/defatted seed meal, and crude myrosinase and glucosinolates with antimicrobial and biofumigation applications. We then demonstrated that both puree and seed oil can serve as effective carbon substrates for cultivation of the oleaginous yeast Yarrowia lipolytica to produce single-cell proteins and high-value recombinant protein products. To use papaya seed oil for culturing $Y$. lipolytica, the concentration of the inhibitory substance benzyl isothiocyanate (BITC) in the oil needs to be minimized. If the culled fruits (and hence seeds) were stored frozen prior to drying, a very high level (>30 mM) of BITC was detected in the oil extracted from the dried seeds. However, if the seeds were not frozen prior to drying, oil from dried papaya seeds contained almost no BITC, and could support vigorous growth of $Y$. lipolytica, with efficient production of a functional nanobody fusion protein at a level similar to that achieved using olive oil. By using both juice and seed lipid, rather than juice alone, $Y$. lipolytica biomass produced per unit papaya more than doubled. As $Y$. lipolytica is amenable to genetic manipulation, and is known as a proficient cell factory with many industrial applications, the papaya waste valorization technology could potentially be extended to produce additional useful products such as biofuel and oleochemicals from Y. lipolytica.
\end{abstract}

Received 28th June 2018
Accepted 27th July 2018
DOI: 10.1039/c8ra05539d
rsc.li/rsc-advances papaya seed lipid as an untapped waste feedstock to provide carbon and energy sources for growing certain microorganisms to produce high-value products.

Yarrowia lipolytica is one such microorganism capable of utilizing lipids as a carbon source. Y. lipolytica is an ideal candidate for utilizing papaya seed oil because it can efficiently metabolize hydrophobic substrates such as alkanes, fatty acids, and fats, and is generally recognized as safe (GRAS). ${ }^{6}$ It is also easy to culture with a short doubling time. Additionally, Y. lipolytica is well characterized especially for lipid and lipase production, ${ }^{7,8}$ and has been shown to be amicable to genetic modification, opening up a wide repertoire of possible applications. ${ }^{9}$ Several studies have looked into bulk industrial products such as biodiesel and citric acid as possible products of $Y$. lipolytica. ${ }^{\mathbf{1 0 , 1 1}}$ In addition, $Y$. lipolytica naturally produces lipases and proteases, which are industrially important enzymes. ${ }^{12,13}$ As an alternative, it is potentially more attractive to produce a high-value product. ${ }^{14}$ With well-developed genetic tools, $Y$. lipolytica can be engineered to produce high levels of recombinant therapeutic proteins, enzymes, or antibodies that can fetch an even higher price.

Before papaya seed oil can be utilized as feedstock for $Y$. lipolytica, however, a potential obstacle that relates to the presence of the inhibitory substance benzyl isothiocyanate (BITC) requires consideration. BITC is a compound, which has
Department of Molecular Biosciences and Bioengineering, University of Hawaii at Manoa, Honolulu, HI 96822, USA. E-mail: wsu@hawaii.edu; Tel: +1808-956-3531

$\dagger$ Electronic supplementary information (ESI) available. See DOI: 10.1039/c8ra05539d 
anti-microbial activity, ${ }^{15,16}$ and is formed when the enzyme myrosinase ( $\beta$-thioglucosidase; EC 3.2.1.147) catalyzes the conversion of the precursor benzyl glucosinolate (BG) into BITC. Normally, myrosinase and BG are spatially separated, with myrosinase largely sequestered in the vacuoles of specialized myrosin (M-) cells, and glucosinolate located in the vacuoles of separate but adjacent "S-cells". ${ }^{17}$ Specifically for papaya seeds, myrosinase activity has been reported in the sarcotestae (fleshy outer coat) and also in the embryo. ${ }^{18}$ When tissue damage occurs, such as during oil extraction, myrosinase and BG come into contact hydrolyzing BG into BITC. It should be noted that while anti-mycotic activity of BITC toward certain yeast species has been reported, ${ }^{\mathbf{1 9 , 2 0}}$ it is unclear as to the effect of BITC on Y. lipolytica.

With the goal to alleviate agricultural waste while simultaneously producing value-added products, the current study investigated the effect of BITC on Y. lipolytica, and ways to simplify papaya fruit waste processing into seeds, peel, and puree, while minimizing the concentrations of BITC in extracted seed oil. The papaya seed oil along with juice were then evaluated for utilization by $Y$. lipolytica to produce a high-value recombinant antibody product.

\section{Experimental}

\section{Papaya fractionation}

Papaya (Carica papaya) fruits (Solo, Rainbow, or Sunrise variety) were used in all experiments and were fully ripe with bright yellow color when used. The whole papaya fruits are fractionated into puree, peel, and a seed fraction that contains mostly undamaged seeds with a minor portion of small peel pieces. We used a stainless steel fruit grinder (useful, UH-FC227) for cider/ wine making to crush whole papaya culls. The resulting crushed papaya chunks were then processed using a hydraulic press (SIA Apple Press, GBP-12) to extract the juice. The remaining seeds and peel pieces collected after juice extraction were then spread out onto a perforated plate sieve $(6.3 \mathrm{~mm}$ aperture size, Endecotts, 200SCF16.0) and seeds (along with some small peel pieces) allowed to pass through while leaving behind bulk of the peel pieces. The seeds were then oven dried at $50{ }^{\circ} \mathrm{C}$ for $48 \mathrm{~h}$.

\section{Soxhlet extraction of papaya seeds}

Dried papaya seeds were weighed, then homogenized using a mortar and pestle. The resulting ground seed powders were placed in a cellulose thimble and the oil was extracted using the Soxhlet extractor, with hexane (Fisher, H292-500) as a solvent. The samples underwent continuous extraction for $3 \mathrm{~h}$. The hexane solvent was removed by rotary evaporation using a Buchi Rotavapor R-114. With most of the hexane removed, residual hexane removal was done by continuous purging of samples with nitrogen. After hexane extraction, the defatted papaya seed ground was further extracted using methanol for $3 \mathrm{~h}$ in the Soxhlet extractor. The methanol solvent was then removed by rotary evaporation, and the residue was reconstituted in $70 \%$ methanol plus $0.1 \%$ trifluoroacetic acid for HPLC analysis of the benzyl glucosinolate (BG) content. $^{21}$

\section{BITC assay}

BITC was measured by using a modified 1,2-benzenedithiol (BDT) cyclocondensation assay. ${ }^{22}$ Briefly, samples containing BITC were pipetted into glass vials and the volume was made up to $1 \mathrm{~mL}$ with a $10 \mathrm{mM}$ potassium phosphate buffer solution at pH 8.5. To these samples, $1 \mathrm{~mL}$ of $4 \mathrm{mM}$ BDT (Sigma, 270865) in methanol (Fisher, A412-4) was added. The samples were capped, briefly vortexed, then placed into a water bath at $65{ }^{\circ} \mathrm{C}$ for $3 \mathrm{~h}$. Then, the reaction mixtures were filtered using a syringe mounted 0.2 $\mu \mathrm{m}$ PES filter (VWR, 28145-501), and analyzed using reverse phase HPLC with a Shimadzu Prominence HPLC system. Samples were run using a Shimadzu Premier C18 column with particle diameter $5 \mu \mathrm{m}$, pore diameter $120 \AA$, length $25 \mathrm{~cm}$, and internal diameter $4.6 \mathrm{~mm}$, with a mobile phase of $80 \%$ HPLC methanol and $20 \%$ HPLC water, isocrati-

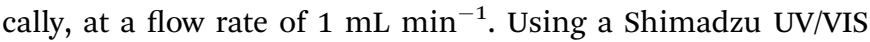
detector, absorbance was measured at a wavelength of $365 \mathrm{~nm}$.

\section{Assay of papaya myrosinase activity}

To determine myrosinase activity, dry ground papaya seeds were extracted with distilled water (papaya myrosinase activity is not affected by drying at $50{ }^{\circ} \mathrm{C}$ for $48 \mathrm{~h}$ ). To the aqueous extract, cold acetone (kept at $-20^{\circ} \mathrm{C}$ ) was added, incubated for $15 \mathrm{~min}$, and centrifuged. The precipitate was redissolved in $0.5 \mathrm{~mL}$ of $33 \mathrm{mM}$ phosphate buffer at $\mathrm{pH} 7.0$ to prepare a crude papaya myrosinase solution. ${ }^{23}$ Myrosinase activity was determined using sinigrin as a glucosinolate substrate, by measuring the rate of decrease in absorbance at $227 \mathrm{~nm}$ due to sinigrin. ${ }^{24}$ One unit of enzyme is defined such that it hydrolyzes one $\mu \mathrm{mol}$ of sinigrin per min. ${ }^{24}$ Sinigrin at $227 \mathrm{~nm}$ has a molar extinction coefficient of $6780 \mathrm{M}^{-1} \mathrm{~cm}^{-1} \cdot{ }^{24}$

\section{Quantification of BG and proximate analysis of defatted papaya seed meal}

Reverse-phase HPLC analysis was used to determine the BG concentration by following the protocol of Rossetto et al., using a Shimadzu Premier C18 column as in BITC measurement. ${ }^{21}$ BG standard was purchased from Cerilliant (Round Rock, TX) (PHY89216). The proximate analysis of the hexane/methanol defatted papaya seed meal was performed by the Agricultural Diagnostic Service Center at the University of Hawaii using standard feed quality analysis methods. ${ }^{25}$

\section{Measurement of total carotenoid contents in papaya seed oil}

To determine the total carotenoid content in papaya oil, $1 \mathrm{~mL}$ of papaya oil was extracted with $2 \mathrm{~mL}$ of $100 \%$ methanol for several times until colorless oil was obtained. The methanol extracts were combined and analyzed for total carotenoid content in a spectrophotometer according to the method of Lichtenthaler and Wellburn. ${ }^{26}$

\section{Constructing a $Y$. lipolytica strain expressing a recombinant nanobody}

Y. lipolytica strain Polg transformed with the pINA69-MNG plasmid for expression of a mCherry tagged anti-GFP 
nanobody fusion protein (termed "mCherry-GFP nanobody" hereinafter) was used throughout this study. ${ }^{27,28}$ Details on plasmid and strain construction can be found in the ESI. $\dagger$

\section{Y. lipolytica culture condition}

Typically the yeast was pre-cultured in $5 \mathrm{~mL}$ of synthetic dextrose (SD) media. The SD media consist of $1.7 \mathrm{~g} \mathrm{~L}^{-1}$ of yeast nitrogen base without ammonium sulfate and amino acids, $5 \mathrm{~g}$ $\mathrm{L}^{-1}$ of ammonium sulfate, $50 \mathrm{mM}$ of potassium phosphate, $1 \mathrm{~g}$ $\mathrm{L}^{-1}$ of yeast extract, and $20 \mathrm{~g} \mathrm{~L}^{-1}$ of glucose. This pre-culture was grown for $24 \mathrm{~h}$ in a shaking incubator at $28{ }^{\circ} \mathrm{C}$ and $200 \mathrm{rpm}$. It was then used to inoculate the oil-based (SO) or puree-based (SP) media that contained $4 \%$ oil $^{11}$ or $25 \%(\mathrm{v} / \mathrm{v})$ puree/juice, respectively, which replaced glucose in the SD media. It is worth noting that it is also feasible to pre-culture in the oil-based media, instead of the SD media. All cultures were grown at $28{ }^{\circ} \mathrm{C}$ at $200 \mathrm{rpm}$.

\section{Culture measurements}

To measure cell growth in the media supplemented with oil (papaya seed oil or olive oil), Y. lipolytica culture was centrifuged at $10000 \times g$ for 3 min and washed with PBS buffer containing $10 \%$ ethanol for three times to remove residual oil from the cell surface. Cells were then resuspended in PBS buffer prior to $\mathrm{OD}_{600}$ measurement. For cultures grown in sugar/juice based media, culture $\mathrm{OD}_{600}$ was taken directly without the washing step. Cell dry weight was determined after oven-drying of filtered cell biomass to constant weight. Residual sugar concentration in the sugar or juice-supplemented media was determined by reducing sugar measurement using the dinitrosalicilic acid (DNS) method. ${ }^{29}$ Residual lipid in the spent medium was determined by extracting using petroleum ether/ chloroform, followed by removing the solvent and measuring the residual lipids gravimetrically according to Papanikolaou et al. $^{30}$

To measure the production of recombinant mCherry-GFP nanobody during cultivation, culture samples were taken periodically and centrifuged to collect the cell pellet. For cells grown in oil-containing media, the cell pellet was first rinsed with PBS buffer containing $10 \%$ ethanol for three times to remove the residual oil on the cell surface. The cells were then resuspended and diluted to $\mathrm{OD}_{600}=0.5$ in PBS buffer for subsequent measurement of mCherry culture fluorescence using a Hitachi F-2500 fluorescence spectrophotometer (excitation and emission at 575 and $610 \mathrm{~nm}$, respectively). The fusion protein concentration was estimated using a fluorescence calibration curve constructed using purified recombinant mCherry. ${ }^{31}$ Besides fluorescence measurements, the amount of recombinant target protein was checked using densitometry analysis of the western blot against His-tagged mCherry standard expressed from Escherichia coli. ${ }^{31}$ For western blots, protein extract was added to SDS-PAGE sample buffer and denatured at $95{ }^{\circ} \mathrm{C}$ for $5 \mathrm{~min}$. After brief centrifugation, denatured protein samples along with a protein ladder were subjected to $12 \%$ SDSPAGE and blotted onto PVDF membranes. After blocking, mouse anti-HisTag (Lamda Biotech, G020) was used as primary antibody, followed by rinsing and then using alkaline phosphatase conjugated goat anti-mouse (Southern Biotech, 101004) as secondary antibody. After rinsing, immunoreactive bands were visualized by NBT/BCIP coupled chromogenic reaction.

\section{GFP-Rsn2 plasmid construction, protein expression, and purification}

Plasmid pET-GR2 encoding a fusion protein comprising GFP and ranaspumin-2 (Rsn2) ${ }^{32}$ was generated by replacing dockerin coding sequence in pETGD $^{33}$ with a Rsn2 coding sequence synthesized by GenScript (Piscataway, NJ). The resulting plasmid was transformed into BL21(DE3) Star ${ }^{\mathrm{TM}}$ chemical competent cells for protein production as described previously. ${ }^{33}$ Details on GFP-Rsn2 expression in E. coli, and subsequent protein purification, can be found in the ESI. $\dagger$

\section{Binding of mCherry-GFP nanobody to GFP-Rsn2 tethered to oil droplets}

To examine the GFP-binding activity of the mCherry-GFP nanobody produced by $Y$. lipolytica, GFP-Rsn2 bound oil droplets were prepared in PBS (with the Rsn2 moiety responsible for being adsorbed to the oil/water interface and displaying GFP on the surface of the oil droplets). To this end, $400 \mu \mathrm{L}$ of olive oil was pipetted into a microfuge tube; $800 \mu \mathrm{L}$ of PBS was then added. Various concentrations of purified GFP-Rsn2 were then added, followed by brief sonication of 3 watts for $30 \mathrm{~s}$, while on ice, using a Q125 ultrasonicator. The emulsified suspension was allowed to incubate at $22{ }^{\circ} \mathrm{C}$ for $30 \mathrm{~min}$. The oil phase was collected via centrifugation at $5000 \mathrm{rpm}$ for $2 \mathrm{~min}$. The oil phase was rinsed twice with DI water to remove any unbound GFPRsn2. Crude protein extract containing mCherry-GFP nanobody was then added, mixed via up-ending the microfuge tube 10 times, and allowed to incubate for $30 \mathrm{~min}$. Unbound mCherry-GFP nanobody fusion protein was removed by rinsing twice. The oil droplets were then examined using an Olympus BX60 fluorescence microscope.

\section{Results and discussion}

\section{BITC effect on Y. lipolytica growth}

It is well documented that papaya seed meal and seed oil contain substances that may have antimicrobial properties..$^{21,23}$ The main active compounds responsible for growth inhibition were believed to be isothiocyanates. BITC is the major isothiocyanate in papaya seeds. Because the literature is limited as to BITC's effect on Y. lipolytica, the first order was to investigate potential inhibitory effects of BITC on this oleaginous yeast. To this end, two series of batch cultures were set up. In one series, Y. lipolytica cells were cultured in the SD media supplemented with differing concentrations of BITC, while a second series of cultures was conducted in the SO media with differing concentrations of BITC (commercial olive oil was used). By measuring the $\mathrm{OD}_{600}$ at the end of a 3 days growth period, the effect of BITC on culture growth was determined.

As shown by both Fig. 1a and b, there was a clear inhibitory effect of BITC on Y. lipolytica, for both sugar- and oil- 
supplemented media. The BITC concentration required for complete growth suppression of Y. lipolytica was approximately 0.020 and $0.377 \mathrm{mM}$, for sugar-based and oil-based media, respectively. The higher BITC tolerance observed in oil-based media was likely a mass transfer effect. As BITC is a hydrophobic compound, it partitions toward the oil and diffuses into the aqueous media where the yeast cells reside. As a result, the effective BITC concentration in the aqueous phase of the SO media should be lower than the overall BITC concentration in the entire SO media.

With BITC confirmed to have inhibitory effects on Y. lipolytica, the next objective was to determine whether BITC levels in raw, extracted papaya seed oil were above or below the inhibitory level presented in Fig. 1. In our initial experiments, papaya seeds were routinely stored at $-20^{\circ} \mathrm{C}$. The seeds were oven dried at $50{ }^{\circ} \mathrm{C}$ for $24 \mathrm{~h}$ prior to oil extraction. It was discovered that the oil extracted from these seeds contains very high levels of BITC (34.96 \pm $2.16 \mathrm{mM}$; Table 1). As the oil-based SO media contain $4 \%$ oil, the BITC concentration in the media would have exceeded the toxic threshold of $0.377 \mathrm{mM}$. Indeed, culture attempts using this papaya seed oil failed to show any growth (Fig. 2).

(a)

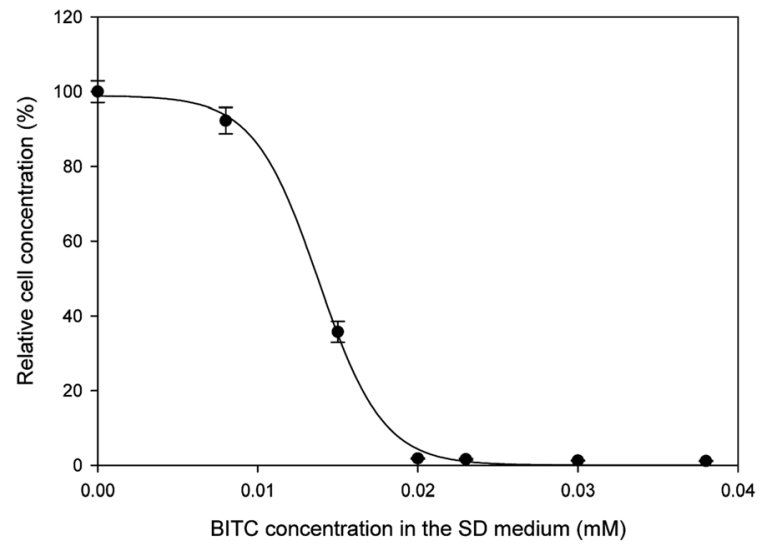

(b)

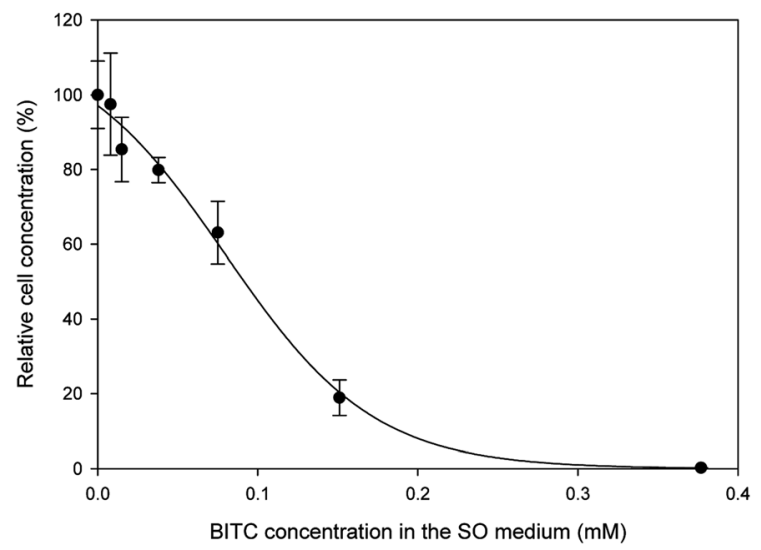

Fig. 1 Concentration-dependent inhibition of BITC on Y. lipolytica cultures. Growth was measured based on $\mathrm{OD}_{600}$ after 3 days of culture. (a) BITC was dissolved in DMSO and added into the SD medium to obtain the target final concentrations; (b) BITC was dissolved in olive oil and incorporated into the SO medium to obtain the target final concentrations. Data represent the mean of three biological replicates $\pm S D$.

\section{Minimizing BITC levels in papaya seed oil by optimizing seed preparation}

Formation of BITC in the papaya seeds results from hydrolysis of BG catalyzed by the enzyme myrosinase. As such, there are a number of pathways can be considered to block the formation of BITC: one may attempt deactivation of myrosinase, removal of the substrate BG, or elimination of moisture from the seeds. To deactivate seed myrosinase, seeds might be subject to thermal treatment with steam, hot air, or hot water, or by microwave heating, before seed oil extraction. To this end, because both BITC and BG are quite resistant to thermal degradation, the thermal treatment was mostly aiming at inactivating the myrosinase. The myrosinase enzymes from different plant species display a wide range of thermal stabilities, some require treatments at a very high temperature, e.g. inactivation of myrosinase in intact rapeseeds required hot water treatment at over $100{ }^{\circ} \mathrm{C}^{34}$ In this case, the high energy cost can be difficult to justify. Another way to minimize the BITC level is to degrade or isolate this compound after it is formed. A recent study reported reduction of BITC levels in raw papaya seed oil by treatment with Aspergillus niger, microwave, or ultraviolet radiations. ${ }^{35}$ While these treatments were reported to lower the BITC they may also affect the quality of the oil with fungal spores or metabolites, or may be prohibitive in large scale, requiring high energy or capital cost.

Comparing to myrosinase deactivation, moisture removal from the seeds prior to oil extraction as a means to block hydrolysis of BG by eliminating water from the reaction appears to be a simpler pathway for controlling BITC levels in the papaya seed oil. Especially, since seeds need to be dried prior to milling and oil extraction. However, as discussed above, by drying frozen papaya seeds to a moisture level below $10 \%$ prior to oil extraction, the resulting oil still contained a significant amount of BITC. We hypothesized that the formation of large ice crystals during the freezing process could alter the internal membrane compartmentalization of the papaya seeds and bring about considerable tissue disruption in the seeds, bringing together BG and myrosinase to generate BITC. As BITC was confined in the seeds, it could not efficiently evaporate during drying, and being lipophilic it ended up in the oil extracted from the seeds. To test this hypothesis, oil was prepared by extracting seeds that were not previously frozen, but rather refrigerated at $4{ }^{\circ} \mathrm{C}$ during storage. These seeds were then dried at either $40^{\circ}$ or $50{ }^{\circ} \mathrm{C}$ for 24 or 48 hours in a gravity convection oven. The moisture content of the seeds under these treatments, as well as the seed oil BITC concentrations are summarized in Table 1 . For the refrigerated seeds, drying at $40{ }^{\circ} \mathrm{C}$ for 48 hours, or at $50{ }^{\circ} \mathrm{C}$ for 24 hours was sufficient to bring the seed oil BITC concentration below $1 \mathrm{mM}$. In culture media supplemented with $4 \%$ oil, the BITC concentration per culture media would be less than $0.04 \mathrm{mM}$ which should allow active growth of $Y$. lipolytica based on data shown in Fig. 1b. This was confirmed as shown in Fig. 2 based on threeday grow-out experiments.

Therefore, a key to preventing high levels of BITC in the extracted seed oil is to avoid damage to the seeds' internal cellular structures, because this cellular damage may cause the 
Table 1 Effect of papaya seed storage (prior to drying) and drying conditions on BITC contents in seed oil ${ }^{a}$

\begin{tabular}{lllrr}
\hline $\begin{array}{l}\text { Storage condition } \\
\text { prior to drying }\end{array}$ & $\begin{array}{l}\text { Drying } \\
\text { temperature }\left({ }^{\circ} \mathrm{C}\right)\end{array}$ & $\begin{array}{l}\text { Drying } \\
\text { duration }(\mathrm{hr})\end{array}$ & $\begin{array}{l}\text { Seed moisture content } \\
\text { after drying }(\% \mathrm{DW})\end{array}$ & $\begin{array}{l}\text { BITC concentration } \\
\text { in seed oil }(\mathrm{mM})\end{array}$ \\
\hline Refrigerated $\left(4^{\circ} \mathrm{C}\right)$ & 40 & 24 & $48.92 \pm 1.74$ & $79.31 \pm 9.38$ \\
Refrigerated $\left(4{ }^{\circ} \mathrm{C}\right)$ & 40 & 48 & $4.82 \pm 0.78$ & $0.94 \pm 0.03$ \\
Refrigerated $\left(4{ }^{\circ} \mathrm{C}\right)$ & 50 & 24 & $4.67 \pm 1.26$ & $0.62 \pm 0.08$ \\
Refrigerated $\left(4{ }^{\circ} \mathrm{C}\right)$ & 50 & 48 & $3.84 \pm 0.78$ & $0.27 \pm 0.04$ \\
Frozen $\left(-20^{\circ} \mathrm{C}\right)$ & 50 & 24 & $3.33 \pm 0.60$ & $34.96 \pm 2.16$
\end{tabular}

${ }^{a}$ Seeds were manually removed from fruits and rinsed before storage.

otherwise segregated BG and myrosinase to react and generate BITC. To this end, storage of culled papaya in freezers prior to drying should be avoided. By simply not freezing the papaya culls and hence seeds prior to drying, we can produce papaya seed oil with essentially no BITC in it. There is no need to rely on costly means (such as thermal deactivation) to deactivate the myrosinase enzyme in the seeds prior to milling and oil extraction, or to detoxify the seed oil by chemical, physical, or biological methods, as reported in other studies. ${ }^{35}$

\section{Low-cost papaya fruit fractionation into multiple value-added products}

In order to utilize the seed oil from papaya fruit waste, it is necessary to establish a practical solution for efficient and costeffective separation of papaya seed, peel, and puree with a sufficiently high throughput to afford scalable operations. In doing so, it is essential not to crush/damage the papaya seeds during the fractionation, otherwise unwanted BITC may result. In this study we established and evaluated a process by repurposing simple, off-the-shelf, fruit processing equipment traditionally used in wine/cider making (consisting of a fruit crusher and a fruit press, ESI Fig. S1†), along with a drying oven and a sieving

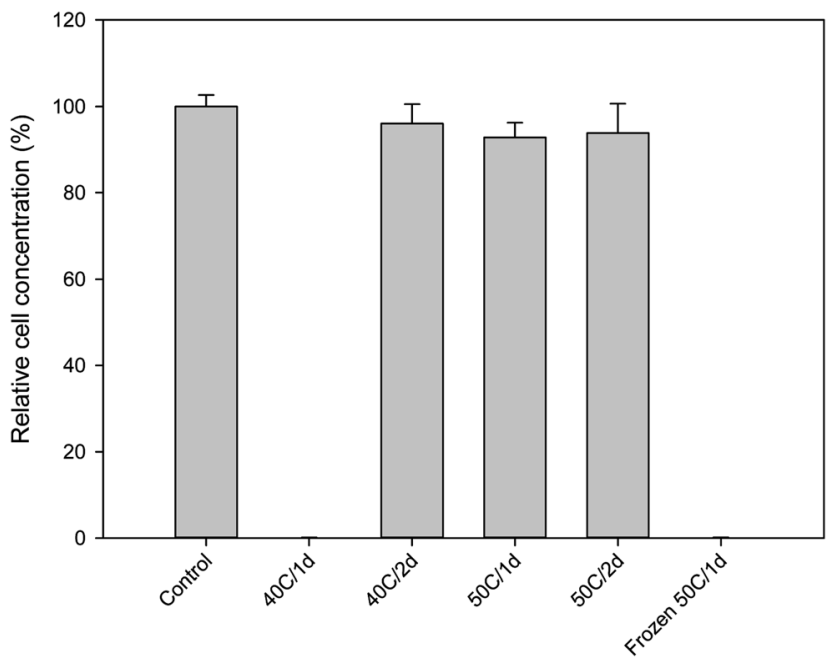

Fig. 2 Growth of $Y$. lipolytica in SO media containing 4\% papaya oil derived from seeds prepared under different storage/drying conditions (cf. Table 1). Growth was measured based on $O D_{600}$ after 3 days of batch culture. Culture grown in $\mathrm{SO}$ media with olive oil was used as control. Data represent the mean of three biological replicates \pm SD. system to achieve efficient fractionation of whole papaya culls into juice pulp, peel, and undamaged seeds. The fractionation scheme and further conversion of juice and seeds into multiple value-added products are summarized in Fig. 3. We first chopped ripe papaya using a fruit crusher, followed by extracting (pressing out) the juice from the chopped ripe fruits using a hydraulic fruit press. The remaining seeds and peel pieces were then separated by sieving using perforated sieve plates with an aperture size that allowed the seeds to pass through while retaining most of the peel pieces. Due to the large gaps between the crushing blades in the fruit crusher (ESI Fig. S1†), seeds are not damaged (and hence no release of the antimicrobial substance such as BITC into the juice) while also allowing the whole fruit to be efficiently processed into chunky pulps (ESI Fig. S1 $\dagger$ ). By eliminating the needs for peeling from initial processing, the proposed fractionation does not require costly and specialized fruit processing machines such as the energy-intensive rotating-drum peeling machine and the fruit pulper/finisher which are required in commercial papaya puree manufacturing. ${ }^{36,37}$ Instead, only very simple and low-cost equipment was needed in the papaya cull fractionation process reported here.

To gauge the performance of the fractionation process, we compared quality of the seed fraction derived from the aforementioned papaya fractionation scheme, with seeds manually separated from the papaya fruits. The results are presented in Table 2. After sieving, the seed fraction contained unbroken seeds at greater than $90 \%$ (on dry weight basis; Table 2). The oil extracted from either of the two papaya seed preparations contained very low levels of BITC and is non-inhibitory to $Y$. lipolytica. Due to the presence of a small portion of peels, seed oil derived from the seed fraction contained a higher total carotenoid content than that in oil derived from seeds only. Upon oil extraction with hexane, the defatted papaya seed meal was further extracted with methanol to obtain a BG-rich extract. The BG content was found to be slightly higher in the seed-only preparation as BG is present at higher levels in seeds than that in peels. ${ }^{21}$ Overall, the seed preparation derived from the simple papaya fractionation process provides an adequate source of seeds for extraction of oil containing very low and nontoxic levels of BITC. Proximate analysis of the seedcake after hexane and methanol extraction indicates a relatively high crude protein content (38.89\% dry mass; ESI Table S1†) while BG was undetectable. To this end, the seed meal might be useful as livestock feeds, though this requires further verifications, and it is beyond the scope of this study. 


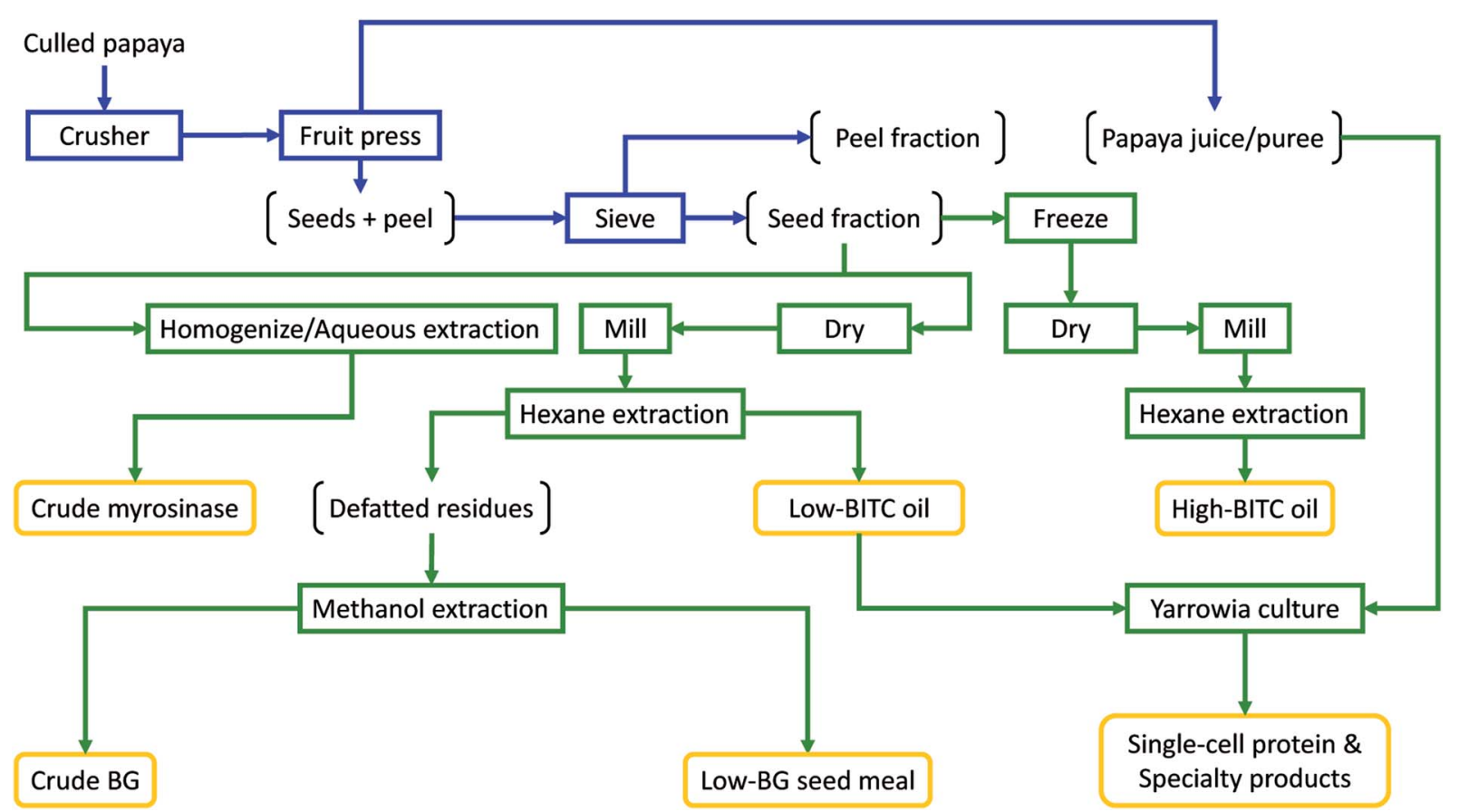

Fig. 3 Process flowchart for low-cost fractionation of culled papaya into peel, puree, and undamaged seeds, followed by processing of the seed fraction to generate crude myrosinase, BITC-rich seed oil, low-BITC seed oil, crude BG, and low-BG seed meal. In addition, papaya juice/puree and low-BITC oil preparation can be used for cultivation of $Y$. lipolytica, or other microorganisms, to produce single-cell-protein and high-value specialty products (such as the nanobody reported in this work). The fruit fractionation portion of the process is highlighted in blue, whereas further processing unit operations are highlighted in green. Potential value-added products are boxed in gold.

Upon fruit fractionation, five different value-added product streams can be generated from the seed fraction (Fig. 3). First, a papaya seed oil that is essentially BITC-free can be produced from non-frozen seeds that are oven-dried and milled, followed by hexane extraction. Second, a BG-rich extract can result when the hexane-defatted seed meal was extracted using methanol. This methanol extraction can also produce the third valueadded product of a refined seed meal with essentially no BG. In this study, we obtained over $30 \mu \mathrm{mol}$ of BG from a gram of dry papaya seeds after hexane and methanol extraction (Table 2), which was on a par with the data reported by Nakamura et al. ${ }^{23}$ The forth product is crude myrosinase prepared from the seeds by aqueous extraction followed by salt or organic solvent (e.g. acetone) precipitation. ${ }^{23}$ Using aqueous extraction and ice-cold acetone precipitation, we obtained 87 units of myrosinase from 100 gram of seeds. During aqueous extraction of the seed powder (Fig. 3), BG was expected to be converted to BITC. However, since BITC is highly hydrophobic, it is not extracted

Table 2 Characterization of the seed fraction derived from the processing scheme depicted in Fig. 3

\begin{tabular}{lcl}
\hline Property & Seed fraction & Seed only $^{a}$ \\
\hline Seed percentage (dry mass basis) & $92.36 \pm 3.13 \%$ & $100 \%$ \\
Peel percentage (dry mass basis) & $7.64 \pm 3.13 \%$ & 0 \\
BG ( $\mu$ mol/g dry seed) & $32.23 \pm 1.74$ & $37.51 \pm 1.15$ \\
BITC content in oil $(\mathrm{mM})$ & $0.22 \pm 0.01$ & $0.18 \pm 0.01$ \\
Total carotenoids in oil $\left(\mu \mathrm{g} \mathrm{mL} \mathrm{mL}^{-1}\right.$ oil) & $35.87 \pm 0.98$ & $7.61 \pm 0.43$
\end{tabular}

${ }^{a}$ Seeds were manually removed from papaya and rinsed prior to drying. into the aqueous solvent and thus is not present in the crude myrosinase product. Finally, the fifth product one may also produce is papaya seed oil with high levels of BITC, by freezing the seeds prior to drying and hexane extraction (Table 1). The BG-rich extract, crude myrosinase, as well as the BITC-rich oil, are potential value-added products that can be used in biofumigation applications for managing soil-borne phytopathogens or in antimicrobial packaging formulations, based on the potent antimicrobial activity of BITC. ${ }^{38,39}$ Utilization of low-BITC oil and papaya puree/juice as a potential carbon source for $Y$. lipolytica was evaluated in this study as discussed below.

\section{Utilization of papaya juice as the sole carbon source for culturing Y. lipolytica}

Papaya puree contains considerable quantities of fermentable monosaccharides, amounting to about $80-90 \mathrm{~g} \mathrm{~L}^{-1}$ of reducing sugars (mainly fructose and glucose, at a ratio of approximately $1: 1$ ) as reported in our previous study and verified in this study. ${ }^{40}$ We modified the SD media by replacing glucose with $25 \%$ papaya juice to prepare the SP media. We also prepared a modified SD media that contained $1 \%$ glucose and $1 \%$ fructose (instead of $2 \%$ glucose). Recombinant $Y$. lipolytica cell growth and nanobody production were evaluated in SP, SD, and modified SD media. As shown in Fig. 4, all three media can support efficient yeast cell growth. It is worth noting that in the SP media, papaya puree was frozen and thawed, followed by centrifugation, and only the supernatant was used in preparing the media. In comparison, when raw papaya puree (without freeze/thaw) was used directly, the yeast culture grew slower and reached a lower 
(a)

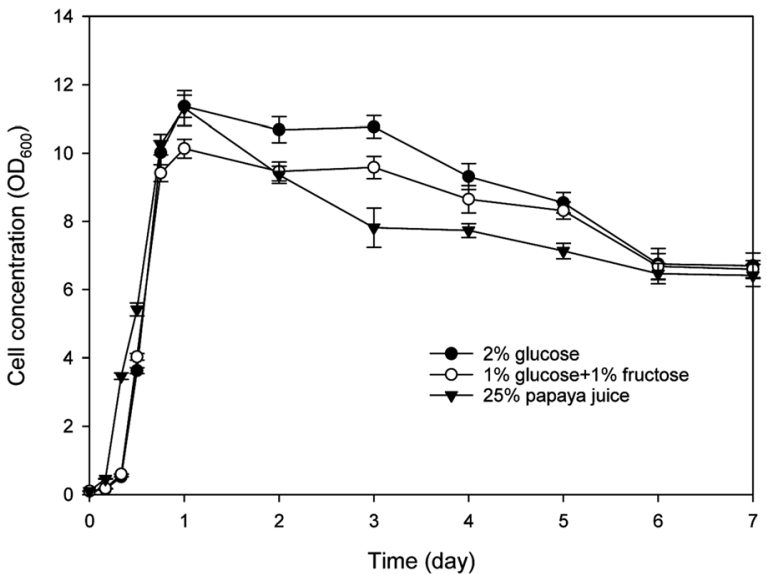

(b)

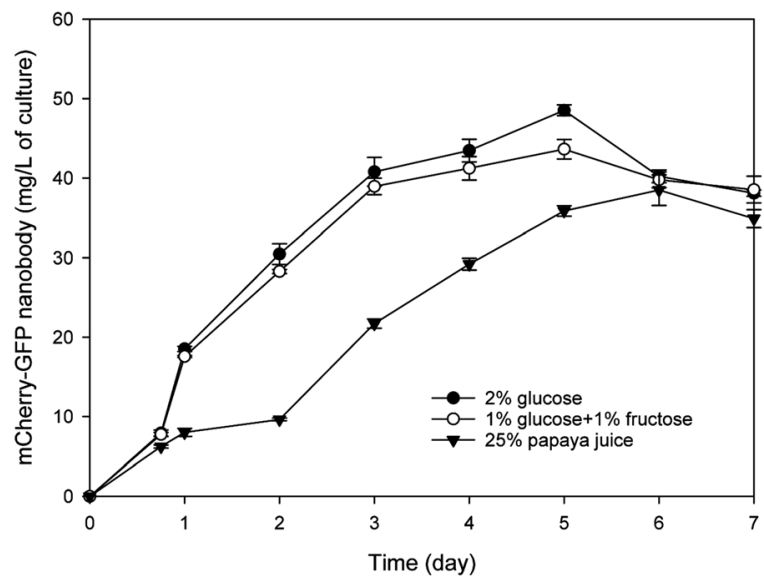

Fig. 4 Evaluation of $Y$. lipolytica cultures utilizing papaya juice/puree (25\%) as carbon source. Culture of the same yeast strain using either $2 \%$ glucose or $1 \%$ glucose plus $1 \%$ fructose serves as control for comparison. Time course of (a) culture growth; and (b) mCherry-GFP nanobody fusion protein production. Data represent the mean of three replicates \pm SD.

final biomass concentration (data not shown). We noted considerable fruit tissue disintegration upon the freezing/ thawing process, which resulted in a much higher amount of clarified juice. Therefore when raw puree was used, much of the sugars may not be readily accessible to the yeast, leading to less efficient growth. Using predetermined cell dry weight $/ \mathrm{OD}_{600}$ correlations, the average yeast biomass yield coefficient on reducing sugars in the SP media was estimated to be $0.467 \mathrm{~g} \mathrm{~g}^{-1}$ on day 1 of the culture while the cell concentration peaked. As for the yeast cultured in the modified SD media, an average yield coefficient of $0.461 \mathrm{~g} \mathrm{~g} \mathrm{~g}^{-1}$ was attained on day 1 of culture. Regarding nanobody production, culture with the SP media displayed a lag compared with the other two cultures but its highest nanobody production still reached about $38 \mathrm{mg} \mathrm{L}^{-1}$ which was just slightly less than that achieved with the modified SD media. Overall, these results corroborate the feasibility of papaya juice as a useful carbon source for culturing $Y$. lipolytica.

\section{Utilization of papaya seed oil as the sole carbon source for culturing Y. lipolytica}

In addition to papaya juice, we evaluated papaya seed oil as the sole carbon source for culturing Y. lipolytica. For comparison, research grade olive oil (Sigma Aldrich, O1514) was also tested in the culture experiment as a control, since Y. lipolytica is known to grow well in olive oil. ${ }^{41}$ Batch cultures of the nanobody-producing $Y$. lipolytica strain were conducted using the SO media containing either papaya or olive oil as the carbon source. As shown in Fig. 5, papaya oil and olive oil gave similar performance in terms of both yeast growth and nanobody production. The highest nanobody production reached 67 and $58 \mathrm{mg} \mathrm{L}^{-1}$ for papaya and olive oil cultures, respectively. The maximum specific growth rate was similar for both papaya and olive oil cultures, at approximately 0.31 per hour. The apparent biomass yield coefficients based on day 3 data were estimated to be 0.367 and $0.341 \mathrm{~g}$ dry cell per $\mathrm{g}$ oil, for papaya and olive oil cultures, respectively. As the fatty acid profile of papaya oil is similar to that of olive oil, this finding came as no surprise. ${ }^{4}$ That said, it is imperative to avoid freezing the seeds prior to drying to prevent accumulation of high levels of BITC in the extracted oil that will inhibit yeast growth. With $4 \%$ initial oil, only a small amount of residual oil $(0.37 \%$ and $0.48 \%$ for papaya and olive oil media, respectively) was detected in the media after 6 days of batch culture, indicating highly efficient uptake and assimilation of fatty acids derived from papaya oil.

(a)

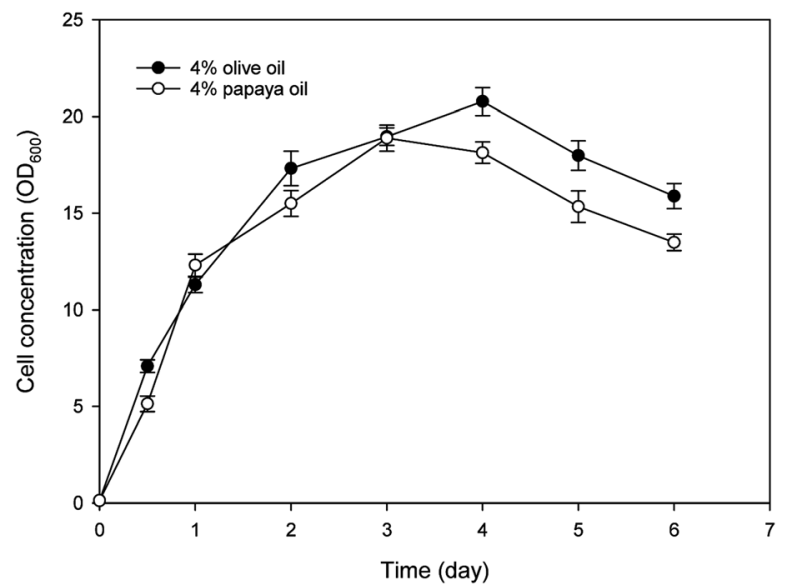

(b)

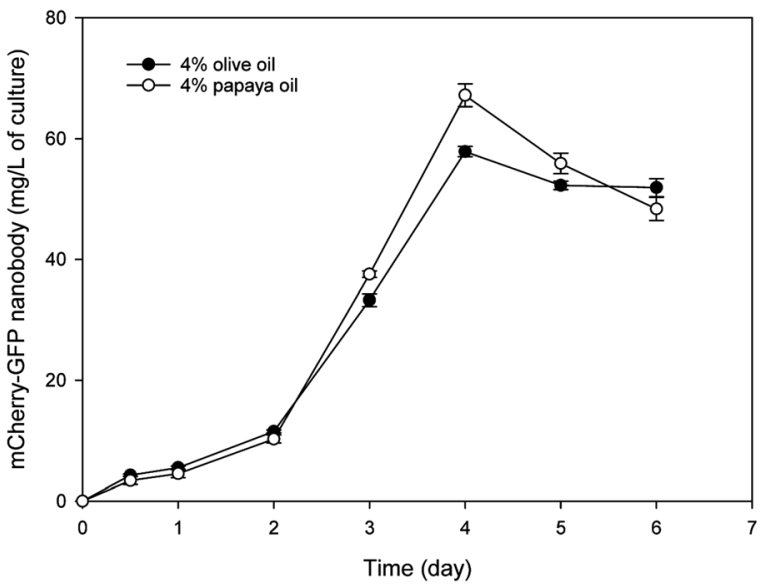

Fig. 5 Evaluation of $Y$. lipolytica cultures utilizing papaya seed oil (4\%) as carbon source. Culture of the same yeast strain using $4 \%$ olive oil serves as control. Time course of (a) culture growth; and (b) mCherryGFP nanobody fusion protein production. Data represent the mean of three replicates \pm SD. 
This low level of residual oil avoids problems that arise from high residual oil levels that could complicate fermentation downstream processing. ${ }^{42}$ The residual oil level may be further reduced by optimizing the initial papaya oil concentration or by fed-batch operation.

Thus far, utilization of papaya waste for microbial production or fermentation has mainly limited to only the juice/puree portion. $^{2,3}$ The present study has demonstrated that papaya seed oil also serves as an effective substrate for microbial production, using the oleaginous yeast $Y$. lipolytica. In fact, by using both juice and seed lipid, rather than juice alone, more than twice the amount of $Y$. lipolytica biomass can be produced per unit papaya. This figure was estimated based on a papaya fruit composition of $60 \%$ puree (containing $8 \%$ sugars), $30 \%$ seeds (containing 25\% lipids), and $10 \%$ peel, along with biomass yield coefficients of 0.467 and $0.367 \mathrm{~g} \mathrm{~g}^{-1}$ for sugars and oil, respectively. $Y$. lipolytica typically utilizes lipid substrates by first hydrolyzing the substrate extracellularly with its very active lipase system, into free fatty acids and glycerol which are then taken up by the yeast. Glycerol enters the central carbon metabolism via phosphorylation to glycerol-3phosphate (G3P) followed by oxidation to dihydroxyacetone phosphate (DHAP) which then enters the glycolytic pathway, ${ }^{43}$ while fatty acids are first activated to fatty acyl-CoA and then oxidized in the peroxisome, used for membrane synthesis, or stored in lipid bodies. ${ }^{6}$ To maximize efficacy of papaya seed oil as a carbon/energy source for $Y$. lipolytica, further studies are underway in our laboratory to better understand assimilation of papaya oil and regulation of fluxes of fatty acid metabolites for cellular activities in $Y$. lipolytica.

Plant oils represent a promising renewable carbon source to support microbial fermentation for producing value-added biobased products. Using plant oil such as rapeseed oil as sole- or co-carbon substrates in large-scale fermentation has been reported for producing antibiotics and polyhydroxyalkanoates (PHAs). ${ }^{44,45}$ Papaya oil represents a unique renewable carbon substrate since it is derived from fruit waste, unlike rapeseed or olive oil. Simple, low-cost processing reported here for producing the oil along with additional valuable coproducts from culled papaya waste, should further improve the economic outlook of papaya oil as an effective renewable carbon source for microbial fermentation.

\section{Functional characterization of Yarrowia-derived nanobody using a novel binding assay}

The $Y$. lipolytica cells from the culture experiments showed a red/pink coloration, and the culture displayed characteristic mCherry fluorescence, indicating the mCherry moiety of the fusion protein functioned properly. Furthermore, the yeast culture extract was analyzed using western blot probed with an anti-His-tag antibody (note a hexa-histidine tag was genetically fused to the C-terminus of the anti-GFP nanobody) as shown in ESI Fig. S2. $\uparrow$ A single band was detected in the western blot with molecular weight closely matching that of the mCherry-GFP nanobody fusion protein $(43.8 \mathrm{kDa})$.

After confirming the expected recombinant protein size, the next task was to determine the functionality of the nanobody portion of the fusion protein for its ability to bind GFP. To accomplish this, first the recombinant protein GFP-Rsn2 was emulsified with olive oils in water to form oil emulsions with GFP-Rsn2 bound to the oil droplets due to the surfactant protein Rsn2 which has strong affinity for the oil/water interface. ${ }^{32}$ The result was the formation of oil droplets with GFP displayed on the surface. Subsequently, incubation of the GFPRsn2 treated oil droplets with yeast cell extracts containing mCherry-GFP nanobody, followed again by collection of the oil phase and washing of unbound fusion protein, resulted in oil droplets that showed fluorescence of both GFP and the bound mCherry (Fig. 6b and c). To validate that the mCherry fluorescence was due to specific binding to GFP and not due to nonspecific adherence to the oil droplets, a negative control was conducted in which olive oil droplets were prepared in the same manner except that no GFP-Rsn2 was added (Fig. 6d-f). This (a)

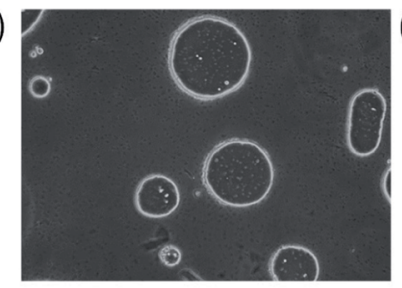

(d)

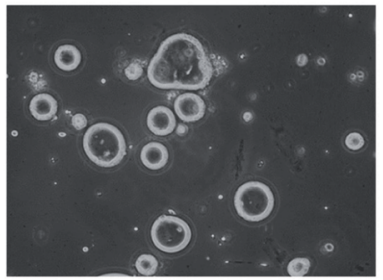

(b)

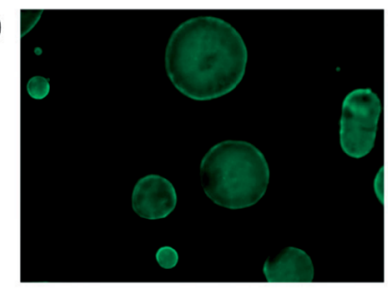

(e)

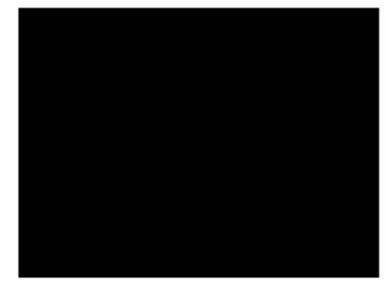

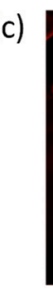

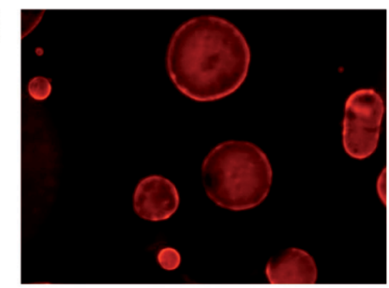

(f)

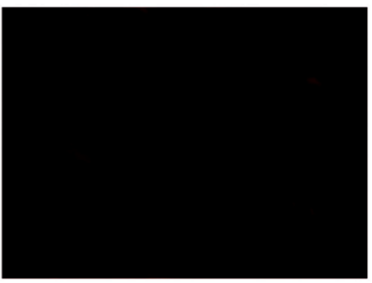

Fig. 6 Functional assay demonstrating specific binding of the mCherry-GFP nanobody to GFP. Two oil emulsions were prepared, one with GFPRsn2 added ( $a, b$ and $c)$, and the other without GFP-Rsn2 ( $d$, e and f). Then, protein extract containing the mCherry-GFP nanobody was incubated with each of the oil emulsions, followed by collection and rinsing to remove excess unbound mCherry-GFP nanobody. Finally, the oil emulsions were examined using fluorescence microscopy with filters to observe GFP (b and e) or mCherry (c and f), and with white light ( $a$ and $d$ ). All pictures taken at $40 \times$ magnification. 
simple assay demonstrated that the GFP nanobody portion of the mCherry-GFP nanobody fusion protein produced by Y. lipolytica growing on papaya seed oil is fully functional. Similar results were found with nanobody derived from cells grown in medium with papaya juice (data not shown). These experiments also illustrated a novel assay platform for detecting specific protein binding, by combining Rsn2-fusion protein and lowcost vegetable oil.

\section{Valorizing papaya waste derived carbon sources with engineered $Y$. lipolytica}

High-value proteins and biodiesel represent two very different pathways to valorize waste papaya seed oil. The price of biodiesel per gram is fractions of a cent. So, while the example recombinant protein in this study (not fully optimized) only achieved a yield of converting approximately $0.17 \%(\mathrm{w} / \mathrm{w})$ of the oil into recombinant protein, the generally much higher unit value of recombinant proteins may compensate for this low yield and make utilizing the papaya seed oil for producing value-added recombinant proteins economically viable. In addition to recombinant proteins and biodiesel, engineered $Y$. lipolytica is known as a proficient cell factory of a wide variety of additional industrial compounds, especially fatty acid-based oleochemicals, many of which have important alternative fuel and energy applications. By establishing a technology that enabled simple production of non-toxic seed oil and juice from papaya waste that is abundant in many regions of the world, and demonstrated efficient assimilation of both of these renewable carbon substrates by Y. lipolytica, it opened doors to new opportunities for sustainable production of high-value bio/ chemicals and, potentially, fuel-like compounds as well. ${ }^{46}$

Logistically, it is envisaged that by using the processing technology developed in this study, small producers can fractionate the papaya culls into puree and unbroken seeds at low cost. The juice can be partially pasteurized and concentrated to reduce volume by simple cooking. The concentrated juice and seeds are densified compared with whole culled fruits and hence easier to transport and store. These materials are then sent off to a centralized processing facility or "centralized biorefinery" to extract seed-derived value-added products including crude myrosinase, BG, BG-free seed meal, and seed oil, while juice and seed oil are further converted into high-value yeast biomass and specialty products. This technology provides a sustainable pathway to valorize papaya waste while solving problems associated with waste disposal.

\section{Conclusions}

In this study, we established a process enabling efficient production of waste papaya derived seed oil that is essentially free of the antimicrobial metabolite BITC. This oil, without any further refining, was shown to be an effective carbon substrate for the industrially important yeast, $Y$. lipolytica, supporting efficient growth with a maximum specific growth rate of about 0.31 per hour, an apparent biomass yield coefficient of $0.367 \mathrm{~g}$ dry cell mass per $\mathrm{g}$ of oil, and high recombinant nanobody production, reaching $67 \mathrm{mg} \mathrm{L}^{-1}$ of culture. Importantly, by using both juice and seed lipid, rather than juice alone (as was done traditionally), Y. lipolytica biomass produced per unit papaya more than doubled. We showed that growth of Y. lipolytica became inhibited by BITC at a low concentration of $0.02 \mathrm{mM}$. However, by avoiding frozen storage of the culled papaya fruit waste, and by minimizing seed breakage during fruit waste fractionation, generation of toxic BITC can be significantly curbed, and the seed oil becomes essentially BITCfree. The papaya fractionation process developed in this study is simple, and with this marked simplicity, this papaya processing scheme is highly amenable to implementation even in resourceconstrained communities. Overall, seed oil from papaya waste represents a promising alternative carbon feedstock, whose conversion by Y. lipolytica opens doors to new opportunities for sustainable production of diverse high-value bio/chemicals.

\section{Conflicts of interest}

The authors declare no conflict of interest.

\section{Acknowledgements}

This work was supported in part by a Specific Cooperative Agreement (SCAno. 58-5320-3-022) with the Daniel K. Inouye U.S. Pacific Agricultural Research Center. The authors acknowledge kind gifts of pINA1269 and the Y. lipolytica Po1g strain from Dr Catherine Madzak of INRA, France.

\section{References}

1 FAO, FAOSTAT: Crops, 2016.

2 W. P. Heller, K. R. Kissinger, T. K. Matsumoto and L. M. Keith, Algal Res., 2015, 12, 156-160.

3 H. Y. Kang, P. Y. Yang, W. G. Dominy and C. S. Lee, Bioresour. Technol., 2010, 101, 7973-7979.

4 E. K. Marfo, O. L. Oke and O. A. Afolabi, Food Chem., 1986, 22, 259-266.

5 F. O. Agunbiade and T. A. Adewole, J. Fuels, 2014, 2014, 1-6. 6 P. Fickers, P. H. Benetti, Y. Wache, A. Marty, S. Mauersberger, M. S. Smit and J. M. Nicaud, FEMS Yeast Res., 2005, 5, 527-543.

7 K. Mathiazhakan, D. Ayed and R. D. Tyagi, Bioresour. Technol., 2016, 221, 234-240.

8 S. Magdouli, T. Guedri, R. Tarek, S. K. Brar and J. F. Blais, Bioresour. Technol., 2017, 243, 57-68.

9 C. Madzak, Appl. Microbiol. Biotechnol., 2015, 99, 4559-4577. 10 S. V. Kamzolova, N. G. Vinokurova, J. N. Lunina, N. F. Zelenkova and I. G. Morgunov, Bioresour. Technol., 2015, 193, 250-255.

11 S. Papamkolaou, M. Galiotou-Panayotou, S. Fakas, M. Komaitis and G. Aggelis, Bioresour. Technol., 2008, 99, 2419-2428.

12 K. N. S. Yadav, M. G. Adsul, K. B. Bastawde, D. D. Jadhav, H. V. Thulasiram and D. V. Gokhale, Bioresour. Technol., 2011, 102, 10663-10670.

13 G. Corzo and S. Revah, Bioresour. Technol., 1999, 70, 173-180. 
14 W. M. Budzianowski, Renewable Sustainable Energy Rev., 2017, 70, 793-804.

15 J. L. Poulsen, A. L. Gimsing, B. A. Halkier, N. Bjarnholt and H. C. B. Hansen, Soil Biol. Biochem., 2008, 40, 135-141.

16 A. Sofrata, E. M. Santangelo, M. Azeem, A. K. Borg-Karlson, A. Gustafsson and K. Putsep, PLoS One, 2011, 6, e23045.

17 E. Andreasson and L. B. Jorgensen, Recent Adv. Phytochem., 2003, 37, 79-99.

18 C. S. Tang, Phytochemistry, 1973, 12, 769-773.

19 M. Galuppo, G. R. De Nicola, R. Iori, P. Dell'Utri, P. Bramanti and E. Mazzon, Molecules, 2013, 18, 14340-14348.

20 E. Kamii and K. Isshiki, J. Food Hyg. Soc. Jpn., 2009, 50, 311314.

21 M. R. M. Rossetto, J. R. O. do Nascimento, E. Purgatto, J. P. Fabi, F. M. Lajolo and B. R. Cordenunsi, J. Agric. Food Chem., 2008, 56, 9592-9599.

22 Y. S. Zhang, Crit. Rev. Food Sci. Nutr., 2012, 52, 525-532.

23 Y. Nakamura, M. Yoshimoto, Y. Murata, Y. Shimoishi, Y. Asai, E. Y. Park, K. Sato and Y. Nakamura, J. Agric. Food Chem., 2007, 55, 4407-4413.

24 S. Palmieri, O. Leoni and R. Iori, Anal. Biochem., 1982, 123, 320-324.

25 I. Cockerel, D. Halliday and D. J. Morgan, Quality Control in the Animal Feedstuffs Manufacturing Industry, Report no. G97, Tropical Products Institute, London, 1975.

26 H. K. Lichtenthaler and A. R. Wellburn, Biochem. Soc. Trans., 1983, 11, 591.

27 C. Madzak, B. Treton and S. Blanchin-Roland, J. Mol. Microbiol. Biotechnol., 2000, 2, 207-216.

28 M. H. Kubala, O. Kovtun, K. Alexandrov and B. M. Collins, Protein Sci., 2010, 19, 2389-2401.

29 G. L. Miller, Anal. Chem., 1959, 31, 426-428.

30 S. Papanikolaou, I. Chevalot, M. Galiotou-Panayotou, M. Komaitis, I. Marc and G. Aggelis, Electron. J. Biotechnol., 2007, 10, 425-435.
31 Z. Han, C. Madzak and W. W. Su, Biotechnol. Bioeng., 2013, 110, 702-710.

32 C. D. Mackenzie, B. O. Smith, A. Meister, A. Blume, X. Zhao, J. R. Lu, M. W. Kennedy and A. Cooper, Biophys. J., 2009, 96, 4984-4992.

33 Z. Han, B. Zhang, Y. E. Wang, Y. Y. Zuo and W. W. Su, Appl. Environ. Microbiol., 2012, 78, 3249-3255.

34 O. A. Okunade, S. K. Ghawi, L. Methven and K. Niranjan, Food Chem., 2015, 187, 485-490.

35 I. S. Afolabi, T. D. Bisi-Adeniyi, T. R. Adedoyin and S. O. Rotimi, J. Food Sci. Technol., 2015, 52, 6475-6483.

36 H. T. Chan, in Fruit juice processing technology, ed. S.Nagy, C. S.Chen and P. E.Shaw, Agscience, Auburndale, FL, 1993, pp. 334-371.

37 E. H. Harter and F. F. Villar, US Pat. 4549479, 1985.

38 P. D. Brown and M. J. Morra, Adv. Agron., 1997, 61, 167-231. 39 D. Savoia, Future Microbiol., 2012, 7, 979-990.

40 M. Provera, Z. Han, B. Y. Liaw and W. W. Su, J. Electrochem. Soc., 2016, 163, A1457-A1459.

41 A. Beopoulos, Z. Mrozova, F. Thevenieau, M. T. Le Dall, I. Hapala, S. Papanikolaou, T. Chardot and J. M. Nicaud, Appl. Environ. Microbiol., 2008, 74, 7779-7789.

42 J. D. Stowell, in Carbon substrates in biotechnology, ed. J. D.Stowell, A. J.Beardsmore, C. W.Keevil and J. R.Woodward, IRL press, Oxford, 1987, pp. 139-159.

43 M. Workman, P. Holt and J. Thykaer, AMB Express, 2013, 3, 58.

44 I. K.-P. Tan and C.-C. Ho, Appl. Microbiol. Biotechnol., 1991, 36, 163-166.

45 G. Jiang, D. J. Hill, M. Kowalczuk, B. Johnston, G. Adamus, V. Irorere and I. Radecka, Int. J. Mol. Sci., 2016, 17, 1157.

46 P. Xu, K. Qiao, W. S. Ahn and G. Stephanopoulos, Proc. Natl. Acad. Sci. U. S. A., 2016, 113, 10848-10853. 